\title{
The Research of Hybrid Genetic Algorithm Based on the Shortest Machine-processing time
}

\author{
Li-Jun ZHONG ${ }^{1, a}$, Le WANG ${ }^{2, b}$ \\ ${ }^{1,2}$ Baicheng Normal University, Baicheng, Jilin Province 137000, P.R. China \\ ajlsbcsfxy@163.com, wawangle00@163.com
}

Keywords: Hybrid genetic algorithm, Machine-processing, Time.

Abstract. A hybrid genetic algorithm is proposed based on the shortest machine-processing time. The searching direction is guided by the both global and local to improve the efficiency of the algorithm. It is applied to solve the engineering problems in a military enterprise, and the results are satisfied.

\section{Introduction}

Job Shop Problem (JSP) is one of the most difficult combinatorial and optimization problems. In recent years, the development of the search methods based on the probabilistic push people to seek solutions to the JSP problem. It has become one of the effective methods to solve this kind of problem, such as Simulation Anneal; Taboo Search; Genetic Algorithm [1]. However, when used the standard genetic algorithm to solve the JSP, the results are often unsatisfactory. Because it is a general optimization algorithm, when to use it to solve problems, it must be combined with the features of the scheduler itself. In this paper, a hybrid genetic algorithm is proposed based on the shortest machine-processing time. The searching direction is guided by the both global and local to improve the efficiency of the algorithm.

\section{Module of GA}

\section{Coding}

We use a coding method based on the workpiece. Coding problem is the key issues of Genetic Algorithms. Genetic algorithm must consider the issue of legitimacy, feasibility, validity and completeness. The same as the JSP. Because of the shortcomings of binary coding can not directly reflect the structure of the demand problem, not easy to develop a special genetic operator, so the use of simple binary encoding methods can not effectively solve the problem JSP [2]. According to the characteristics of the problem, we use a coding method based on the workpiece. Using this method, we can find the corresponding coding of the decoding; replacement arrangement of any workpiece could represent feasible schedule, also the length of encoded is less than the binary encoding [3].

\section{Genetic operators}

Choose operator

Select the operator using roulette method. Because the non-negative requirements of roulette method, and in order to enhance the differences between individuals, the paper uses the scaling method of sigma truncation to determine the fitness of individuals. The formula is [4]:

$$
\mathrm{F}=(\text { score-ave })+\mathrm{c} * \mathrm{dev}
$$

Where score is original objective function value, ave is average of population; dev is the variance of population.

Cross operator

Cross using partial matching method. The method is not directly cross between the two parents, but two offspring by exchanging their corresponding position genes to achieve cross [5]. The advantage is that no illegal solution. The efficiency of the program is improved. 
Mutation Operator

To make the algorithm can search the optimal solution in a wider feasible solution, we use the following method: Random draw two points, then swap genes according to the probability. This will ensure the diversity of individuals, while retaining the portion of the gene.

\section{The process of hybrid genetic algorithm}

Step 1: Generate initial population. Cross using partial matching method.

Step 2: Calculate the fitness value. Use the scaling method of sigma truncation to determine the fitness of individuals.

Step 3: Determine whether reached the number of iterations or whether the genetic is stabilized. If reached, output the optimal scheduling solution. Otherwise, carry out the fourth step.

Step 4: Genetic manipulation.

(1) As the method described above, select the parent individuals which required crossing.

(2) According probability to determine whether two parent needs cross.

(3) Variation manipulation

(4) Back to Step3, determine whether the termination condition is satisfied, satisfied is terminated; otherwise Step 2.

\section{The use of genetic algorithms to solve scheduling problems}

\section{Example}

Population size is 40 ; crossover probability is 0.9 ; mutation probability of 0.01 . In algorithm design, the 13 kinds of workpieces to be processed are numbered 1-13. Used machine also label, shown in Table 1.

Table 1 Processing machines and number

\begin{tabular}{|c|c|c|}
\hline Name & code & number \\
\hline Five-axis horizontal machining center & M1 & 1 \\
\hline Turning Centers & M2 & 2 \\
\hline Axis horizontal machining center & M3 & 3 \\
\hline CNC milling machine & M 4 & 4 \\
\hline CNC milling machine & M 5 & 5 \\
\hline CNC milling machine & M 6 & 6 \\
\hline Peripheral processing equipment & M 7 & 7 \\
\hline Horizontal Machining Center & M 8 & 8 \\
\hline Heat Treatment Equipment & M 9 & 9 \\
\hline Fluorescent devices & M 10 & 10 \\
\hline Vertical machining center & M 11 & 11 \\
\hline & & 12 \\
\hline & M 1, M 3, M 8 & 13 \\
\hline & M 2, M 3 & 14 \\
\hline & M 1, M 3 & 15 \\
\hline & M2, M3, M 11 & 16 \\
\hline & M3 M8 & 17 \\
\hline
\end{tabular}

Among them, the number 12 means a workpiece maybe produced by the machine tools coded "M1, M2, M3, M4, M5, M6, M8, and M10". The number 13 means "M1, M3, and M8". The same to the number 14 , number 15, number 16 and number17. The order matrix of machine is shown in table 2. 
Table 2 The order matrix of machine

\begin{tabular}{|c|c|c|c|c|c|c|c|c|c|}
\hline $\begin{array}{c}\text { Part } \\
\text { number }\end{array}$ & \multicolumn{9}{|c|}{ Machine number } \\
\hline 1 & 12 & 5555 & & & & & & & \\
\hline 2 & 12 & 12 & 12 & 12 & 5555 & & & & \\
\hline 3 & 13 & 13 & 13 & 9 & 13 & 13 & 13 & 5555 & \\
\hline 4 & 14 & 14 & 14 & 14 & 14 & 14 & 14 & 10 & \\
\hline 5 & 1 & 10 & 1 & 5555 & & & & & \\
\hline 6 & 8 & 5555 & & & & & & & \\
\hline 7 & 12 & 9 & 7 & 12 & 15 & 5555 & & & \\
\hline 8 & 13 & 13 & 5555 & & & & & & \\
\hline 9 & 12 & 12 & 9 & 12 & 12 & 12 & 12 & 10 & 5555 \\
\hline 10 & 12 & 5555 & & & & & & & \\
\hline 11 & 12 & 5555 & & & & & & & \\
\hline 12 & 12 & 5555 & & & & & & & \\
\hline 13 & 16 & 9 & 7 & 16 & 8 & 8 & 17 & 17 & 5555 \\
\hline
\end{tabular}

Table 2 is a workpiece's order matrix of machine; it is stored in a txt file. The benefit is easy to change data when the machines or parts changed. The program runs, reads data from a file and stored in the corresponding machine matrix. "5555" means new line. Workpiece machining time matrix is shown in table 3.

Table 3 Workpiece machining time matrix

\begin{tabular}{|c|c|c|c|c|c|c|c|c|c|}
\hline $\begin{array}{c}\text { Part } \\
\text { number }\end{array}$ & \multicolumn{9}{|c|}{ Step } \\
\hline 1 & 20 & 5555 & & & & & & & \\
\hline 2 & 80 & 20 & 240 & 160 & 5555 & & & & \\
\hline 3 & 24 & 24 & 48 & 48 & 24 & 24 & 48 & 5555 & \\
\hline 4 & 60 & 40 & 27 & 27 & 60 & 47 & 140 & 32 & 5555 \\
\hline 5 & 12 & 32 & 192 & 5555 & & & & & \\
\hline 6 & 200 & 5555 & & & & & & & \\
\hline 7 & 80 & 48 & 112 & 240 & 40 & 5555 & & & \\
\hline 8 & 15 & 15 & 5555 & & & & & & \\
\hline 9 & 32 & 32 & 48 & 24 & 8 & 24 & 24 & 32 & 5555 \\
\hline 10 & 160 & 5555 & & & & & & & \\
\hline 11 & 160 & 5555 & & & & & & & \\
\hline 12 & 72 & 5555 & & & & & & & \\
\hline 13 & 80 & 48 & 112 & 80 & 20 & 120 & 20 & 20 & 5555 \\
\hline
\end{tabular}

Table 3 is a workpiece machining time matrix. The initial data is also stored in a txt file. "5555" also means new line.

\section{The results}

Standard genetic algorithm

SGA start from a group scheduling solution, and explore a wide range of solutions by using a variety of genetic manipulation. Then the evaluation function evaluate each solution, good population reserved 
and in order to continue looking for the best scheduling solution. This approach has the ability to find the optimal solution, but in a random manner, the search process aimlessly, unable to guarantee the quality of the solution. The Gantt of standard genetic algorithm is shown in Figure 1.

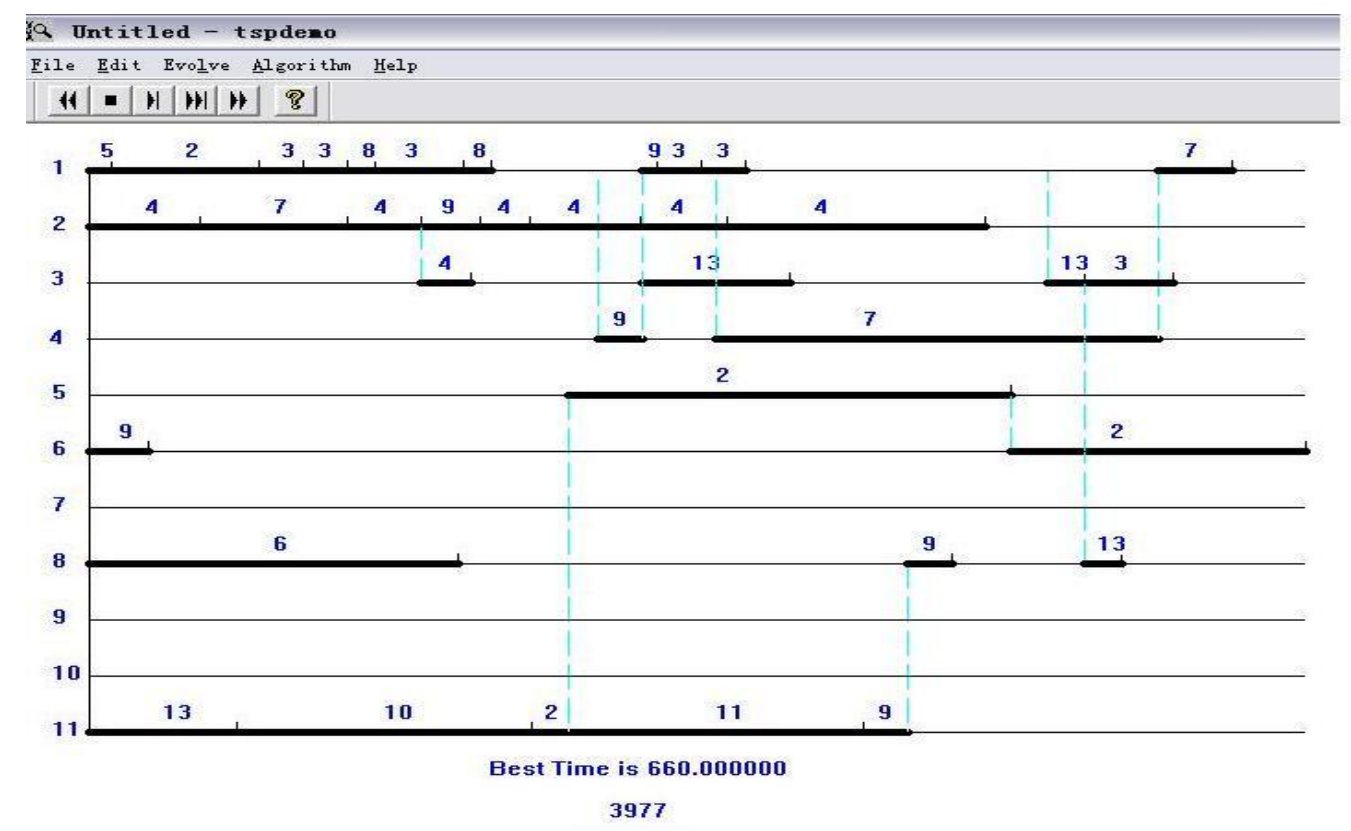

Figure 1 The Gantt chart of standard genetic algorithm

Figure 1 is the Gantt chart of standard genetic algorithm which is run 3977 generations. The time is 660 hours. The result is not satisfactory.

Hybrid Genetic Algorithm

In this paper, the machining matrix is generated by the method of shortest processing time. Every time the program access only a step, and sort function is called once for all machines work in ascending order according to the length of time. Then compare that can be scheduled according to the procedure and the sorting machine, determine the machine which every step of the workpiece used. Until complete the processing matrix. The Gantt of hybrid genetic algorithm is shown in Figure 2.

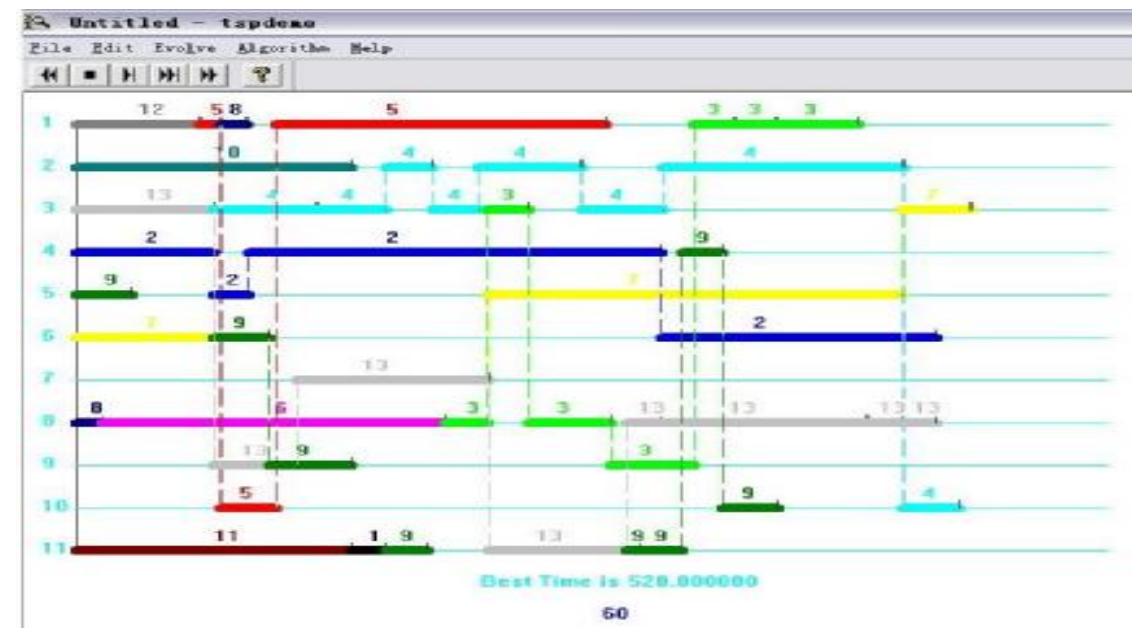

Figure 2 The Gantt chart of hybrid genetic algorithm

Figure 2 is the Gantt chart of hybrid genetic algorithm which is run 60 generations. The time is 520 hours. The results compared with the standard genetic algorithm are better than SGA. The generations reduced, the results achieved better. 


\section{Conclusions}

The HGA which integration the shortest machining concepts into algorithms, reduced the search space of GA, and improved the efficiency of the algorithm. Examples show that the hybrid genetic algorithm has good advantage and effectiveness.

\section{References}

[1] Ling Wang, Workshop scheduling and GA, Qinghua University Press, 2003.

[2] De Jong K A. Genetic algorithm: a 25 year perspective, computational intelligence imitating Life, The Institute of Electrical and Engineers, Inc.1994

[3] Srinivas M, Patnaik K M. Adaptive probabilities of crossover and mutation in genetic algorithms, IEEE Transactions on Systems, Man and Cybernetics.1994

[4] Yi Zeng, Study on job shop scheduling based on modified genetic algorithm, Machinery Design and Manufacure, 7(2011)180-182.

[5] Ling Wang, Advances in Job shop Scheduling Based on Genetic Algorithm, Control and Decision. 16(2001)641-646. 